\title{
Irreversible electroporation of locally recurrent pancreatic cancer with 5-year survival
}

\author{
Alyssa M Deiters ${ }^{1}$, Minia Hellan ${ }^{1,2}$, Rebecca M Tuttle ${ }^{1,2}$ and James R Ouellette ${ }^{1,2 *}$ \\ ${ }^{1}$ Department of Surgery, Wright State University Boonshoft School of Medicine, Dayton, Ohio, USA \\ ${ }^{2}$ Division of Surgical Oncology, Wright State University Boonshoft School of Medicine, Dayton, Ohio, USA
}

\begin{abstract}
Background: Isolated local recurrence after surgical resection for pancreatic cancer is rare. Current common therapies for recurrence include chemotherapy or chemoradiation. There are few, if any, reports regarding surgical resection of local pancreatic cancer recurrence. This is the first report of using irreversible electroporation (IRE) to treat a pancreatic adenocarcinoma local recurrence and represents a true 5-year survivor and possible cure.

Case report: A 50-year-old female initially presented with $2.5 \mathrm{~cm}$ mass in the uncinate process of the pancreas suspicious for malignancy. Preoperative endoscopic guided fine needle aspirate (FNA) confirmed adenocarcinoma. She underwent pylorus preserving pancreaticoduodenectomy with the finding of stage II pancreatic cancer with complete resection after neoadjuvant chemotherapy. One year later she developed recurrent abdominal pain and restaging scans confirmed a new 2.5 $\mathrm{cm}$ lesion in the retroperitoneum. Positron emission tomography (PET) scan confirmed local recurrence. No metastatic disease was identified. She subsequently underwent chemotherapy and radiation therapy with persistent mass lesion present. After a period of stability, she was taken for laparotomy with IRE of the entire retroperitoneal tumor, followed by adjuvant chemotherapy. She has been closely monitored with computed tomography (CT) scans and tumor markers. Her most recent scan confirmed complete resolution of the lesion. She is now over 6 years from pancreaticoduodenectomy with no additional signs of pancreatic cancer recurrence.

Conclusions: Patients with isolated recurrent pancreatic cancer are uncommon and have limited treatment options. IRE is currently used for locally-advanced, unresectable pancreatic cancer with promising results. This case serves as an example that IRE can contribute to enhanced long term survival for patients with pancreatic adenocarcinoma, including those with isolated local recurrence.
\end{abstract}

\section{Introduction}

Pancreatic cancer is the fourth leading cause of cancer death in men and women in the United States [1]. Although there are only about 50,000 new cases of pancreatic cancer cases a year, the mortality rate is very high. Five-year survival rates in the US for local, regional and metastatic disease are $29 \%, 11 \%$, and $3 \%$ [1]. Local recurrence after surgical resection is around $30 \%$, and there are limited modalities for treatment once the patient reaches this point [2]. Chemotherapy or chemoradiation has become standard of care after pancreatic cancer local recurrences. Surgical resection was also recently shown to have a favorable survival outcome in resectable isolated local recurrent pancreatic cancer, but further studies are warranted [3].

Advances in ablative therapies over the past two decades allow patients to have another possible option of treatment for locally advanced pancreatic cancer (LAPC) or locally recurrent pancreatic cancer. Thermal ablative therapies include radiofrequency ablation (RFA) and microwave ablation (MWA), while non-thermal ablative therapies include irreversible electroporation (IRE). Thermal ablation is a poor treatment modality for tumors located close to vital structures, such as vessels, bowel, or ducts, which is why the IRE technology has gained popularity with pancreatic head tumors. This emerging technology uses electrodes to deliver short, pulsed currents to create nanoscopic pores in cell membranes of tumor cells, which causes cell death or apoptosis. IRE has been proven to be feasible for tumor ablation in the liver, pancreas and prostate, while efficacy in organs such as the lung and kidney have not been substantially proven [4].
This is the first reported case of a patient with locally recurrent pancreatic cancer after chemotherapy and surgical resection who was subsequently treated with multi-modality therapy, including irreversible electroporation (NanoKnife; AngioDynamics; Queensbury, NY, USA) and is now a true 5-year survivor, and possible cure.

\section{Case presentation}

This is a 50 year-old Caucasian female with past medical history of gastroesophageal reflux and tobacco use (17 pack years) who presented initially in 2010 with complaints of epigastric and right upper quadrant pain radiating to her back. Initial work up included esophagogastroduodenoscopy (EGD), right upper quadrant ultrasound, and HIDA scan which were normal, except for esophagitis and gastritis. Due to worsening pain, she underwent an abdominal CT scan in March 2010 which showed a $2.5 \mathrm{~cm}$ ill-defined mass located in the pancreatic uncinate process (Figure 1). Her exam was unremarkable. Laboratory results showed normal liver enzymes, bilirubin, amylase, lipase, but an elevated CA 19-9 of 451. Patient was enrolled in the clinical

Correspondence to: James R Ouellette, Department of Surgery, WSU Boonshoft School of Medicine, 3535 Southern Blvd., Kettering, OH 45429 USA; Tel: 9373958686; E-mail: James.ouellette@wright.edu

Key words: pancreatic cancer, irreversible electroporation, recurrence

Received: October 30, 2017; Accepted: November 23, 2017; Published: November 27, 2017 


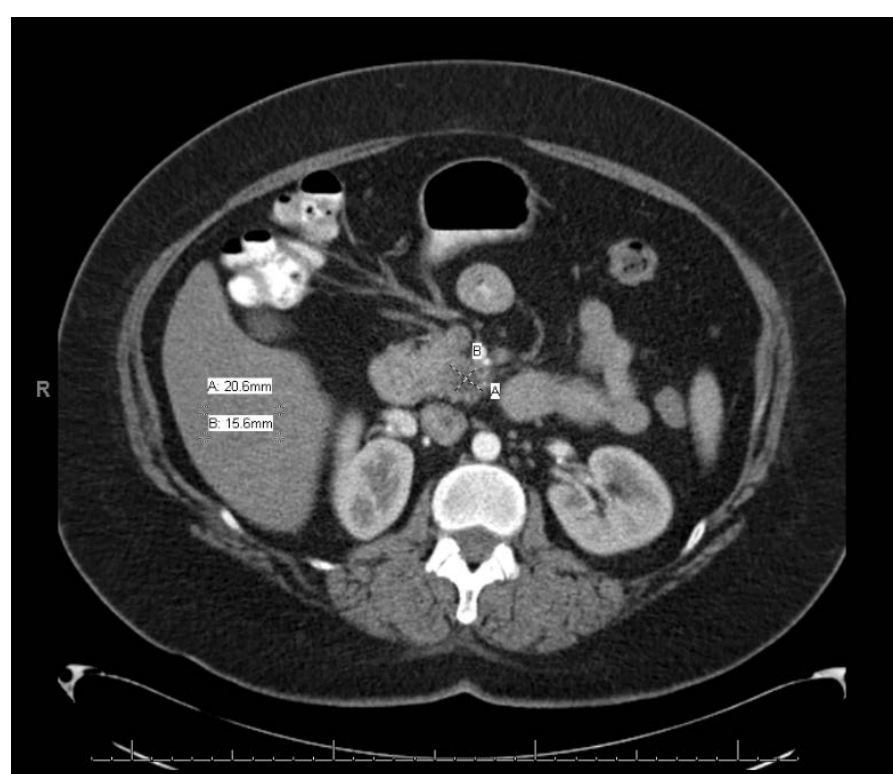

Figure 1. CT imaging of primary pancreatic adenocarcinoma (March 2010)

trial ACOSOG Z5041 where she received 3 cycles of neoadjuvant chemotherapy (gemcitabine and erlotinib). This was a phase II trial that addresses the benefit of erlotinib as an adjunct to gemcitabine during the perioperative setting for resectable pancreatic cancer.

The patient underwent a pylorus preserving Whipple procedure in July 2010. Intraoperatively there was no evidence of metastatic disease. The tumor was firm and palpable in the uncinate process and extended up to the fatty tissue in the retroperitoneum next to the superior mesenteric artery (SMA). The SMA was ultimately skeletonized to resect the tumor with $\mathrm{R} 0$ resection. Tumor was $1 \mathrm{~cm}$ in size with grade 3 chemotherapy effect, $<10 \%$ viable tumor cells. No vascular invasion was noted. Pathology confirmed 16 negative lymph nodes, and all margins were negative. Three cycles of postoperative chemotherapy per trial protocol. Follow up consisted of physical exam, CT scans, labs every 3-4 months for the first year, and every 4-6 months after.

She did well for the next year, but developed recurrent abdominal pain in May 2011. Abdominal CT scan demonstrated a new lesion in the retroperitoneum at previously placed clip (Figure 2). Endoscopic ultrasound (EUS) with biopsy showed a $2.5 \mathrm{~cm}$ mass, but was inaccessible for biopsy. PET scan confirmed significant uptake with SUV of 9.2 in this area only. She then completed four more cycles of chemotherapy (gemcitabine and oxaliplatin), and received radiation. On repeat abdominal CT scan in October 2011, the lesion decreased in size to $2 \mathrm{~cm}$, but did not resolve.

Due to residual mass lesion present in December 2011, an exploratory laparotomy with IRE of the area of recurrent pancreatic cancer was planned. A solitary small nodule, about $2 \mathrm{~cm}$ in size, was palpated and confirmed by ultrasound to be on the SMA anterior to the IVC and was penetrated by the main branches of the SMV. The nodule was deemed unresectable. The decision was then made to ablate the nodule with the Nanoknife. Three needles were used to create a triangular field. In determining optimal tissue ablation in this patient, we placed the needles $1.5 \mathrm{~cm}$ apart and performed $1.5 \mathrm{~cm}$ exposure and applied 90 pulses per cycle between 2 needles. Ultrasound confirmed placement and changes of the nodule within placement. There was no replacement of the needles, but a single pull back of the needles to allow adequate coverage of the entire lesion.
Follow up imaging showed post-operative inflammatory changes which are expected after IRE. Repeat imaging was performed at 1 month, 3 months, and then at 3-4 month intervals over the next 2 years with eventful resolution of the mass lesion. Most recent imaging is showing in (Figure 3 ). Tumor markers are also checked regularly and returned to normal and currently remain within normal ranges. She continues to be followed routinely.

\section{Discussion}

Ablative therapies (thermal and nonthermal) have emerged as innovative treatment options for locally advanced pancreatic cancer (LAPC), but are also able to be utilized for locally recurrent disease. Thermal ablation techniques have significant limitations due to risk of damaging vital structures. Radiofrequency ablation (RFA) causes tissue destruction through high frequency alternating currents generating high local temperatures $\left(>40^{\circ} \mathrm{C}\right)$ which cause a coagulative necrosis [5]. Risks of RFA therapy include thermal injury to nearby vasculature, bowel and biliary tree which leads to complications such as portal vein thrombosis, duodenal injury and gastrointestinal bleeding [6,7]. Microwave ablation (MWA) is another treatment modality for LAPC. A generator uses microwave energy via an antenna to heat the desired tissue and cause coagulative necrosis. The frequency range of MWA compared to RFA allows a better predictable ablation volume [6]. A case series with 15 patients with LAPC concluded no major procedure related morbidity and mortality. Most common complications were minor and include mild pancreatitis, asymptomatic hyperamylasemia, pancreatic ascites, and minor bleeding [8]. Further long-term studies are needed to determine survival benefit of MWA despite early results of low patient morbidity in this small patient population.

In contrast, nonthermal ablative techniques such as irreversible electroporation (IRE) could be used for pancreatic tumors that are near vessels and vital structures, which made it an ideal choice for our patient with a tumor closely located near the IVC, portal vein, or SMA [5]. The device works by applying short, pulsed, high-voltage electrical currents across a cell which leads to non-reversible poration, the formation of nanopores in the cell membrane, which causes apoptosis of the cell [9]. Only the lipid bilayer of the cell membrane is affected with IRE, which is in contrast to thermal ablation where all the molecules are affected [6]. IRE can be used percutaneously with imaging guidance, laparoscopically, or through an open approach. Probes are positioned

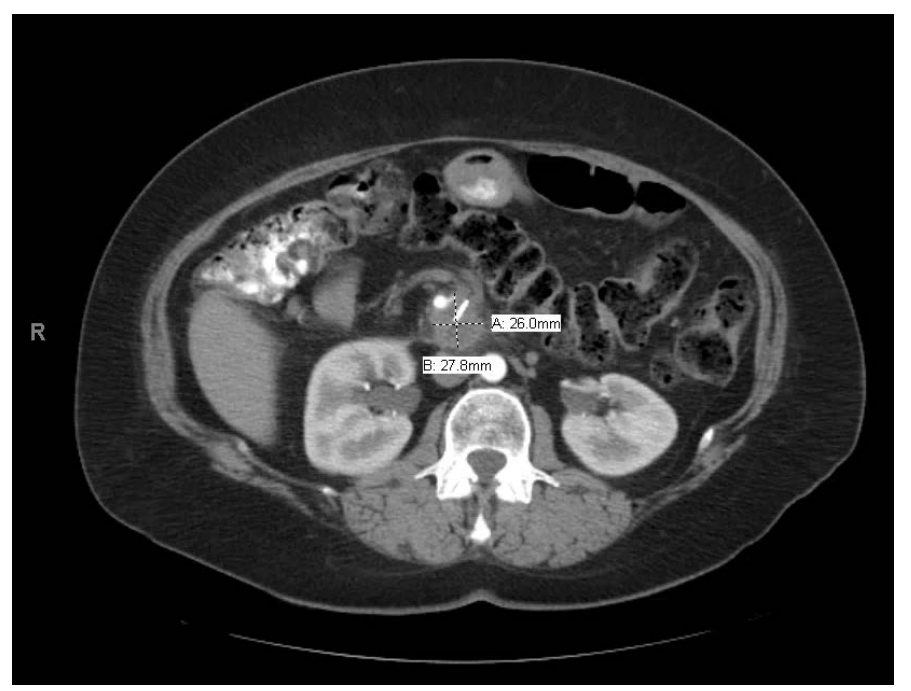

Figure 2. Recurrence of pancreatic adenocarcinoma (May 2011) 

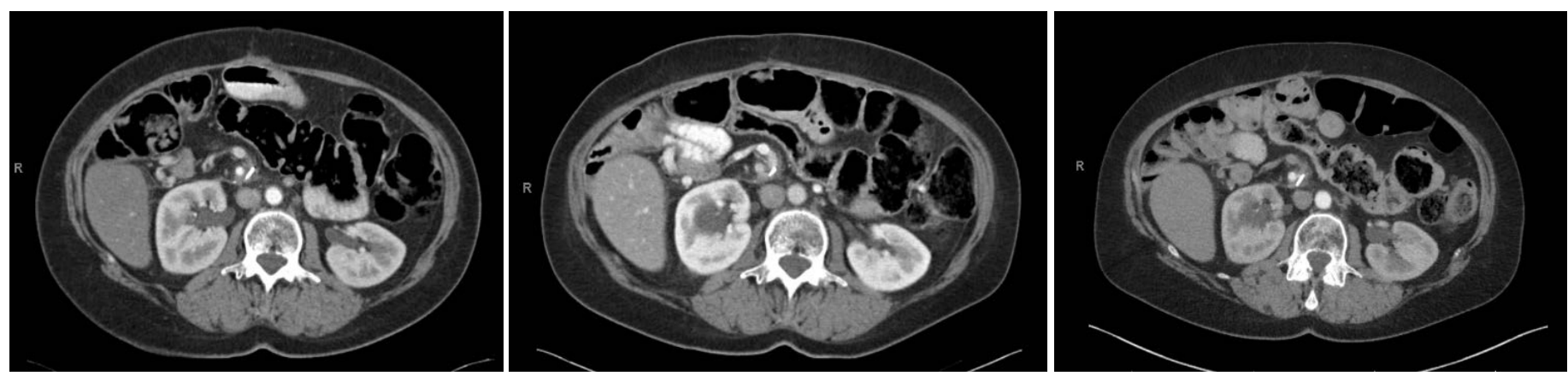

Figure 3. Most recent follow up imaging $(2013,2015,2017)$ after IRE therapy

parallel to one another at an ideal spacing of $2 \mathrm{~cm}$ from one another to ensure complete electroporation of the target, however pancreatic tissue frequently requires adjustment of exposure and spacing to accommodate tissue penetration [10].

Due to the mechanism of action targeting the cell membrane only, IRE has been successfully demonstrated safe ablation in liver porcine models without negatively affecting nearby blood vessels and bile ducts [11]. A pilot study used IRE ablation in vivo in the pancreas of porcine models to successfully show histological evidence of irreversible ablation as soon as 2 hours post procedure without signs of pancreatitis or pancreatic duct injury [12]. The unique characteristics of not injuring nearby blood vessels or ducts allows IRE to be the modality of choice to treat tumors within close approximation to vital structures in comparison to other ablation techniques. There is also no loss of heat secondary to convection ("heat sink effect") which leads to incomplete ablation as documented with thermal ablation techniques [9].

Limitations of IRE include the need for general anesthesia and tumor size. General anesthesia with complete muscle paralysis is necessary to counteract the stimulation of skeletal muscles with the high voltage delivered to the probes and to prevent muscle contraction from moving the position of the probes [9]. The optimal size of locally advanced pancreatic cancer is less than $3 \mathrm{~cm}$ for new users, and less than $4 \mathrm{~cm}$ for experienced users [10]. Our patient's presentation with recurrent pancreatic cancer was $2 \mathrm{~cm}$ in size and within these limits. Contraindications for IRE include history of cardiac arrhythmia, recent myocardial infarction, and nearby vicinity to devices with metal parts (such as metal biliary stent). IRE pulse delivery is synchronized with the patient's electrocardiogram to minimize risk of cardiac event. Arrhythmias are contraindicated because the pulses are unable to be synchronized with the R-waves [9]. Metallic stents may conduct the electrical current and deflect the energy causing damage to surrounding structures or incomplete ablation [13]. Pre-operative removal of these stents is advisable.

Extensive research about LAPC and IRE has been performed by Martin et al. A prospective evaluation of long term effects of IRE on liver and pancreatic locally advanced carcinoma showed that the IRE attributable morbidity rate was $13.3 \%$, with only $4.19 \%$ high grade complications seen, such as bleeding, biliary complications and DVT/ PE being most common. Although pancreatic lesions were more likely to have complications than liver lesions, the safety profile was similar to other studies. Local recurrence rate was $23 \%$, with median time for pancreatic recurrence was 16 months [14].

Previous treatment options for patients with unresectable or locallyrecurrent pancreatic cancer were solely limited to chemotherapy or chemoradiation therapy. The comparison of IRE patients who received at least 4 months of neoadjuvant chemo to standard treatment of adjuvant chemotherapy showed a statistically significant improvement in local progression-free survival, distant progression-free survival and overall survival [15]. Due to IRE only targeting the local disease, pre-operative and post-operative chemotherapy with or without chemoradiation is still administered to attempt to control potential undetected distant lesions as was performed in our patient.

Martin et al proposed a clinical usage algorithm for IRE in patients with locally advanced stage III pancreatic adenocarcinoma, which may be able to be applied to locally recurrent pancreatic tumors as well. They recommend precise staging with triple phase CT scan and diagnostic laparoscopy with peritoneal washings to accurately discern stage III local disease with a sub-radiologically occult stage IV patient [10]. At least four months of induction chemotherapy is then completed, with repeat imaging after chemotherapy to ensure there is no progression of disease. At this time, definitive local therapy or palliative procedures may be performed, which is where IRE may be applicable.

Uses for IRE technology in pancreatic cancer currently include therapy for unresectable locally advanced pancreatic cancer, and margin accentuation during surgical resection [10]. The application of this technology can potentially be expanded to locally-recurrent pancreatic cancer near vital structures if the patient is found to be a suitable candidate.

\section{Conclusion}

Most research for IRE in pancreatic cancer involves its use with unresectable stage III locally advanced disease. There is a paucity of data regarding its use with locally recurrent pancreatic cancer after surgical resection. Therapy in these patients is inherently limited. Though conclusions are obviously limited based on this single case report, IRE can be considered in this patient population in combination with the standard treatment of chemotherapy or chemoradiation. Further studies or series will be needed before we can identify a true survival advantage.

\section{References}

1. American Cancer Society (2017). Cancer Facts and Figures.

2. Groot VP, van Santvoort HC, Rombouts SJE, Hagendoorn J, Borel Rinkes, et al. (2017) Systematic review on the treatment of isolated local recurrence of pancreatic cancer after surgery; re-resection, chemoradiotherapy and SBRT. $H P B$ 19: 83-92. [Crossref]

3. Strobel O, Hartwig W, Hackert T, Hinz U, Berens V, et al. (2013) Re-resection for isolated local recurrence of pancreatic cancer is feasible, safe, and associated with encouraging survival. Ann Surg Oncol 20: 964-972. [Crossref]

4. Savic LJ, Chapiro J, Hamm B, Gebauer B, Collettini F (2016) Irreversible Electroporation in Interventional Oncology: Where We Stand and Where We Go. Fortschr Rontgenstr 188: 735-745. [Crossref]

5. Keane MG, Bramis K, Pereira SP, Fusai GK (2014) Systematic Review of Novel Ablative Methods in Locally Advanced Pancreatic Cancer. World J Gastroenterol 20 : 2267-2278. [Crossref] 
6. Linecker M, Pfammatter T, Kambakamba P, DeOliveira ML (2016) Ablation strategies for locally advanced pancreatic cancer. Dig Surg 33: 351-359. [Crossref]

7. Girelli R, Frigerio I, Giardino A, Regi P, Gobbo S, et al. (2013) Results of 100 pancreatic radiofrequency ablations in the context of a multimodal strategy for stage III ductal adenocarcinoma. Langenbeck's Arch Surg 398: 63-69. [Crossref]

8. Lygidakis NJ, Sharma SK, Papastratis P, Zivanovic V, Kefalourous H, et al. (2007) Microwave ablation in locally advanced pancreatic carcinoma--a new look. Hepatogastroenterology 54: 1305-1310. [Crossref]

9. Govindarajan N (2015) Irreversible Electroporation. Semin Intervent Radiol 32: 349355. [Crossref]

10. Robert MCG (2015) Use of Irreversible Electroporation in Unresectable Pancreatic Cancer. Hepatobiliary Surg Nutr 4: 211-215. [Crossref]
11. Boris R, Onik G, Mikus P (2007) Irreversible Electroporation: A New Ablation Modality-Clinical Implications. Technol Cancer Res Treat 6: 37-48. [Crossref]

12. Charpentier KP, Wolf F, Noble L, Winn B, Resnick M, et al. (2010) Irreversible Electroporation of the Pancreas in Swine: A Pilot Study. HPB 12: 348-351. [Crossref]

13. Efishat M, Wolfgang CL, Weiss MJ (2015) Stage III pancreatic cancer and the role of irreversible electroporation. $B M J 350$ : h521. [Crossref]

14. Martin RC, Philips P, Ellis S, Hayes D, Bagla S (2014) Irreversible Electroporation of Unresectable Soft Tissue Tumors with Vascular Invasion: Effective Palliation. BMC Cancer 14: 540. [Crossref]

15. Martin RC 2nd, McFarland K, Ellis S, Velanovich V (2013) Irreversible electroporation in locally advanced pancreatic cancer; potential improved overall survival. Ann Surg Oncol 20: S443-S449. [Crossref]

Copyright: (C2017 Deiters AM. This is an open-access article distributed under the terms of the Creative Commons Attribution License, which permits unrestricted use, distribution, and reproduction in any medium, provided the original author and source are credited. 